\title{
Considerations on the design of diffractive optical needles
}

\author{
Rosario Martínez-Herrero \\ Departamento de Óptica \\ Universidad Complutense de Madrid \\ Madrid, Spain \\ r.m-h@fis.ucm.es \\ Artur Carnicer \\ Departament de Física Aplicada \\ Universitat de Barcelona \\ Barcelona, Spain \\ artur.carnicer@ub.edu
}

\author{
David Maluenda \\ Centro Nacional de Biotecnología \\ Consejo Superior de Investigaciones Científicas \\ Madrid, Spain \\ dmaluenda@cnb.csic.es
}

\author{
Ignasi Juvells \\ Departament de Física Aplicada \\ Universitat de Barcelona \\ Barcelona, Spain \\ ignasi.juvells@ub.edu
}

\begin{abstract}
According to intensity consideration along the propagation axis, we derived mathematical conditions for a family of modulating functions able to produce needles of arbitrary length, sub-wavelength width and almost constant irradiance. These functions are derived by introducing mathematical constrains on the on-axis power content. As a result, the modulating distribution is continuous with no jumps within or at the edge of the pupil. Numerical results suggests that the proposed approach is suitable for producing high quality needles.

Index Terms-Light needles, High Numerical Aperture optical systems, Polarization.
\end{abstract}

\section{INTRODUCTION}

Most designs proposed for generating optical needles are based on the utilization of binary or ternary ring-geometry modulation functions [1]-[6]. Nevertheless, diffractive needles produced by means of high numerical aperture objective lenses usually display remarkable distortions and lack of quality. To avoid this behavior, several authors developed alternative techniques to avoid the use of bulky diffractive optical elements. In particular, super-oscillatory lenses have been made using both spatial light modulators and metamaterials [7]-[10]. Interestingly, polarization takes a key role in the calculation of optical needles, as pointed out in [11]-[14]. In this communication we discuss our recent developments on the design of diffractive optical needles [15].

\section{BACKGROUND}

The electric field $\mathbf{E}$ at the focal area is described by the Richards-Wolf integral [16]

$$
\begin{aligned}
\mathbf{E}(r, \phi, z)=A \int_{0}^{\theta_{0}} \int_{0}^{2 \pi} & \mathbf{E}_{0}(\theta, \varphi) \exp (i k r \sin \theta \cos (\phi-\varphi)) \\
& \exp (-i k z \cos \theta) \sin \theta \mathrm{d} \theta \mathrm{d} \varphi
\end{aligned}
$$

$k$ is the wave number, $(r, \phi, z)$ are the cylindrical coordinates at the focal area, $\theta$ and $\varphi$ are the polar and the azimuthal angles

R.M.H. and A.C. acknowledges support from Ministerio de Economía y Competitividad (MINECO) grant number FIS2016-75147-C3-1-P. at the Gaussian sphere of reference and $A$ is a constant. The Numerical Aperture (NA) of the focusing lens is $\mathrm{NA}=\sin \theta_{0}$ where $\theta_{0}=\max \{\theta\}$ is the semi-aperture angle.

$\mathbf{E}_{0}$ is the vector angular spectrum described by

$$
\mathbf{E}_{0}=\sqrt{\cos \theta}\left(\left(\mathbf{E}_{s} \cdot \mathbf{e}_{1}\right) \mathbf{e}_{1}+\left(\mathbf{E}_{s} \cdot \mathbf{e}_{2}^{\prime}\right) \mathbf{e}_{2}\right),
$$

where $\mathbf{e}_{1}$ and $\mathbf{e}_{2}$ are unit orthogonal vectors on the azimuthal and radial directions and $\mathbf{e}_{2}^{\prime}$ is the projection of $\mathbf{e}_{2}$ on the entrance pupil plane:

$$
\begin{array}{r}
\mathbf{e}_{1}=(-\sin \varphi, \cos \varphi, 0) \\
\mathbf{e}_{2}=(\cos \theta \cos \varphi, \cos \theta \sin \varphi, \sin \theta) \\
\mathbf{e}^{\prime}{ }_{2}=(\cos \varphi, \sin \varphi, 0) .
\end{array}
$$

$\mathbf{E}_{s}$ describes the transversal beam distribution at the entrance pupil of the optical system, namely

$$
\mathbf{E}_{s}(\theta, \varphi)=g(\theta) h(\theta) \mathbf{p}(\varphi) .
$$

$h(\theta)$ is a modulation function tailored for producing light needles and $\mathbf{p}(\varphi)$ ) describes the input polarization pattern. Without loss of generality, the illuminating beam profile $g(\theta)$ is considered Gaussian, i.e.:

$$
\mathbf{E}_{s}=\frac{1}{\sqrt{2}} \exp \left(-\frac{\sin ^{2} \theta}{f_{0}^{2} \sin ^{2} \theta_{0}}\right)\left(h_{x}(\theta, \varphi) \mathbf{e}_{x}+h_{y}(\theta, \varphi) \mathbf{e}_{y}\right),
$$

where $\mathbf{e}_{x}=(1,0,0), \mathbf{e}_{y}=(0,1,0)$ and $f_{0}$ is the filling factor.

\section{Optical NeEdLe Design}

In this paper we propose a modulation function $h(\theta)$ able to produce long light needles with sub-wavelength width and almost constant irradiance. First, we introduce vector function $\tilde{\mathbf{F}}$, which is related to the electric field on the optical axis $\mathbf{E}(0, z)$ by means of the following equation:

$$
\mathbf{E}(0, z)=\int_{0}^{\theta_{0}} \tilde{\mathbf{F}}(\theta) e^{-i k z \cos \theta} \sin \theta \mathrm{d} \theta
$$


with

$$
\begin{aligned}
\tilde{\mathbf{F}}(\theta) & =A \int_{0}^{2 \pi} \mathbf{E}_{0}(\theta, \varphi) \mathrm{d} \varphi= \\
& A \int_{0}^{2 \pi} \sqrt{\cos \theta}\left(\left(\mathbf{E}_{s} \cdot \mathbf{e}_{1}\right) \mathbf{e}_{1}+\left(\mathbf{E}_{s} \cdot \mathbf{e}_{2}^{\prime}\right) \mathbf{e}_{2}\right) \mathrm{d} \varphi .
\end{aligned}
$$

The modulation function $h(\theta)$ is selected in such a way that $\tilde{\mathbf{F}}(\cos \theta)$ is continuous and presents a zero jump at the entrance pupil edge, i.e. $\tilde{\mathbf{F}}\left(\cos \theta_{0}\right)=0$. Moreover, it is required that $\tilde{\mathbf{F}}$ displays large derivative values. If both conditions holds, it can be demonstrated that the length of the light distribution $2 L$ on the $z$-axis that encloses at least the $75 \%$ of the on-axis power content is

$$
2 L=\frac{2 \lambda}{\pi}\left[\frac{\int_{\alpha_{0}}^{1}\left|\frac{\mathrm{d} \tilde{\mathbf{F}}(\alpha)}{\mathrm{d} \alpha}\right|^{2} \mathrm{~d} \alpha}{\int_{\alpha_{0}}^{1}|\tilde{\mathbf{F}}(\alpha)|^{2} \mathrm{~d} \alpha}\right]^{\frac{1}{2}} .
$$

Among all possible modulation distributions $h(\theta)$ that produces $\tilde{\mathbf{F}}(\cos \theta)$ functions with the desired mathematical properties, we propose the following one:

$$
h(\theta)=N \operatorname{sinc}\left(2 \pi N \frac{\cos \theta-\alpha(m)}{1-\alpha_{0}}\right) \sin \theta
$$

where $\alpha(m)$ is

$$
\alpha(m)=\frac{m}{2 N}\left(1-\alpha_{0}\right)+\alpha_{0},
$$

and $\operatorname{sinc}(x)$ is the unnormalized cardinal sine function; $N$ and $m$ are natural numbers $(0<m<2 N)$ and factor $\alpha(m)$ is related to the transverse width. Note that $h(0)=h\left(\theta_{0}\right)=0$.

\section{NUMERICAL RESULTS}

In order to test the proposed modulation function $h(\theta)$, we simulated the behavior of a optical needle with the following parameters: $N=m=7, \mathrm{NA}=0.85$. In this case, the input beam $\mathbf{E}_{s}$ is assumed to be radially polarized. The filling factor of the Gaussian illuminating beam is $f_{0}=1.5$.

The simulation is performed considering an optical system able to produce beams with arbitrary complex amplitude and polarization [17], [18]. Lack of flatness and codification errors in the liquid crystal devices are also taken into account.

The produced needle is about $35 \lambda$ long with sub-wavelength width. The three images on the top show the transverse light distribution at the planes indicated by the arrows. Interestingly, the axial intensity displays an almost constant irradiance.

\section{CONCLUDING REMARKS}

Summarizing, we proposed a new family of modulation functions for producing diffractive needles with arbitrary polarization and almost constant irradiance. These function are derived by introducing mathematical constrains on the onaxis power content. As a result, the modulating distribution is continuous with no jumps within or at the edge of the pupil. To illustrate their behavior, we simulated a $35 \lambda$-long needle in realistic conditions.
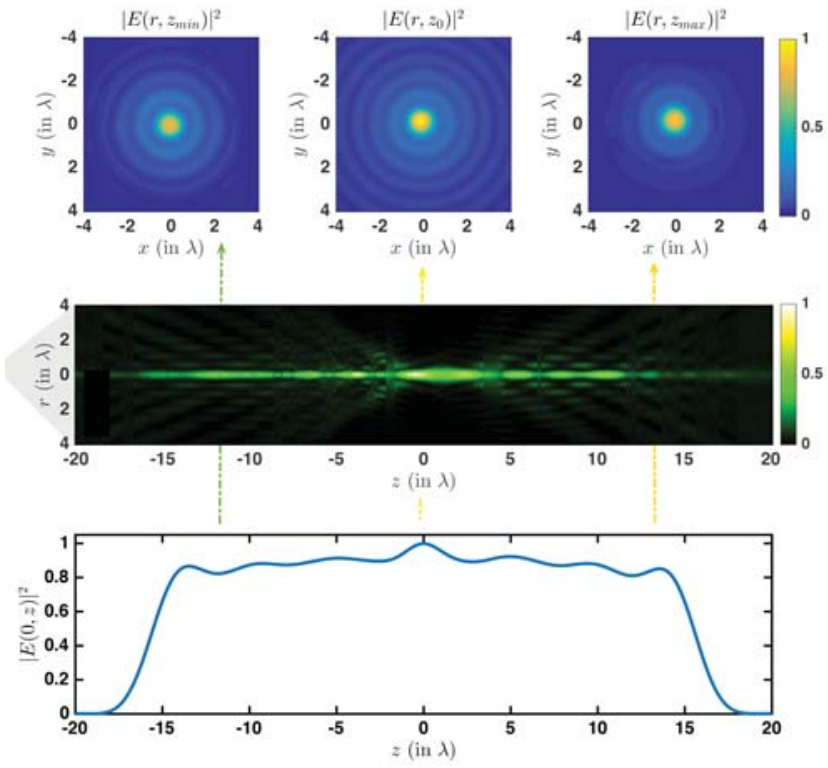

Fig. 1. Simulation of a radially polarized optical needle. $N=m=7$, NA $=0.85, f_{0}=1.5$

\section{REFERENCES}

[1] Wang, H., Shi, L., Lukyanchuk, B., Sheppard, C. J. R. \& Chong, C. T. Creation of a needle of longitudinally polarized light in vacuum using binary optics. Nat. Photon. 2, 501-505 (2008).

[2] Rajesh, K., Jaroszewicz, Z. \& Anbarasan, P. Improvement of lens axicons performance for longitudinally polarized beam generation by adding a dedicated phase transmittance. Opt. Express 18, 26799-26805 (2010).

[3] Sheppard, C. J. R. \& Mehta, S. Three-level filter for increased depth of focus and bessel beam generation. Opt. Express 20, 27212-27221 (2012).

[4] Zha, Y., Wei, J., Wang, H. \& Gan, F. Creation of an ultra-long depth of focus super-resolution longitudinally polarized beam with a ternary optical element. J. Opt. 15, 075703 (2013).

[5] Sheppard, C. J. R. Optimization of pupil filters for maximal signal concentration factor. Opt. Lett. 40, 550-553 (2015).

[6] Xing, J., Kim, J. \& Yoo, H. Design and fabrication of an optical probe with a phase filter for extended depth of focus. Opt. Express 24, 10371044 (2016).

[7] Rogers, E. T. et al. Super-oscillatory optical needle. Appl. Phys. Let. 102, 031108 (2013).

[8] Yuan, G. et al. Planar super-oscillatory lens for sub-diffraction optical needles at violet wavelengths. Sci. Rep. 4, 6333 (2014).

[9] Veysi, M., Guclu, C., Boyraz, O. \& Capolino, F. Reflective metasurface lens with an elongated needle-shaped focus. J. Opt. Soc. Am. B 34, 374-382 (2017).

[10] Yu, Y., Huang, H., Zhou, M. \& Zhan, Q. Creation of a multi-segmented optical needle with prescribed length and spacing using the radiation pattern from a sectional-uniform line source. Sci. Rep. 7, 10708 (2017).

[11] Kitamura, K., Sakai, K. \& Noda, S. Sub-wavelength focal spot with long depth of focus generated by radially polarized, narrow-width annular beam. Opt. Express 18, 4518-4525 (2010).

[12] Lin, J., Yin, K., Li, Y. \& Tan, J. Achievement of longitudinally polarized focusing with long focal depth by amplitude modulation. Opt. Lett. 36, 1185-1187 (2011).

[13] Dehez, H., April, A. \& Piché, M. Needles of longitudinally polarized light: guidelines for minimum spot size and tunable axial extent. Opt. Express 20, 14891-14905 (2012).

[14] Qin, F. et al. Shaping a subwavelength needle with ultra-long focal length by focusing azimuthally polarized light. Sci. Rep. 5, 9977 (2015). 
[15] Martínez-Herrero, R., Maluenda, D., Juvells, I. \& Carnicer, A. Shaping a subwavelength needle with ultra-long focal length by focusing azimuthally polarized light. Sci. Rep. 8, 2657 (2018).

[16] Richards, B. \& Wolf, E. Electromagnetic diffraction in optical systems. II. structure of the image field in an aplanatic system. P. Roy. Soc. London A Mat. 253, 358-379 (1959).

[17] Maluenda, D., Juvells, I., Martínez-Herrero, R. \& Carnicer, A. Reconfigurable beams with arbitrary polarization and shape distributions at a given plane. Opt. Express 21, 5432-5439 (2013).

[18] Maluenda, D., Martínez-Herrero, R., Juvells, I. \& Carnicer, A. Synthesis of highly focused fields with circular polarization at any transverse plane. Opt. Express 22, 6859-6867 (2014). 